\title{
Evolution of Fusarium graminearum A3/5 grown in a glucose-limited chemostat culture at a slow dilution rate
}

\author{
Marilyn G. Wiebe, ${ }^{1}$ Geoffrey D. Robson, ${ }^{1}$ Stephen G. Oliver ${ }^{2}$ and \\ Anthony P. J. Trinci ${ }^{1}$
}

Author for correspondence: Marilyn G. Wiebe. Tel: +44 61275 3918. Fax: +44 612755656.

1 School of Biological Sciences, 1.800 Stopford Building, University of Manchester, Manchester M13 9PT, UK

2 Manchester Biotechnology Centre, University of Manchester Institute of Science and Technology, PO Box 88, Manchester M60 1QD, UK
The evolution of Fusarium graminearum $A 3 / 5$ grown in a glucose-limited chemostat at a dilution rate of $0.05 \mathrm{~h}^{-1}$ (doubling time of $13.9 \mathrm{~h}$ ) was followed for $957 \mathrm{~h}$ or 69 generations. Periodic selection of advantageous mutants was monitored in the culture by determining increases and decreases in the concentration of cycloheximide-resistant macroconidia in the population. Six peaks in the concentration of cycloheximide-resistant macroconidia were observed representing five adaptive changes in the population; on average, an adaptive change occurred once every $148 \pm 22 \mathrm{~h}$ (mean \pm SE). The selection coefficient of strains present at the start of each increase in the concentration of cycloheximide-resistant macroconidia (i.e. after the establishment of a new advantageous strain) was determined relative to $A 3 / 5$ and was found to increase progressively with time. When grown at a dilution rate of $0.05 \mathrm{~h}^{-1}$, the strain (A28-S) isolated from the last adaptive peak had a selection coefficient of $0.023 \mathrm{~h}^{-1}$ relative to A3/5, but A28-S lost its selective advantage when grown at a dilution rate of about $0.11 \mathrm{~h}^{-1}$ and was at a selective disadvantage when grown at a dilution rate higher than $0.11 \mathrm{~h}^{-1}$. The $K_{\mathrm{m}}$ value $(12 \pm 5 \mu \mathrm{M})$ for uptake of glucose by A28-S was significantly lower than that for A3/5. The spontaneous mutation rate from cycloheximide sensitivity to cycloheximide resistance was estimated to be $1.8( \pm 0.2) \times 10^{-6} \mathrm{~h}^{-1}$ or $2.5 \times 10^{-5}$ generation $^{-1}$. The culture initially contained about $1 \times 10^{6}$ macroconidia $\mathrm{ml}^{-1}$ but this decreased with time until, at about $800 \mathrm{~h}$, the culture contained only about $1 \times 10^{4}$ macroconidia $\mathrm{ml}^{-1}$. No highly branched (colonial) mutants were observed in glucose-limited cultures at dilution rates of $0.05 \mathrm{~h}^{-1}$, even though the evolution of the population was followed for a further $1345 \mathrm{~h}$ in a second chemostat, making a total evolutionary period of $2207 \mathrm{~h}$ or 159 generations.

Keywords: Fusarium graminearum A3/5, chemostat culture, $K_{\mathrm{s}}$ mutants, selection coefficient, periodic selection

\section{INTRODUCTION}

When an organism is grown in a chemostat, the relationship between its specific growth rate $(\mu)$ and the concentration of the growth limiting substrate $(S)$ is described by the equation (Monod, 1942):

$$
\mu=\frac{\mu_{\max } S}{\left(S+K_{\mathrm{s}}\right)}
$$

where $\mu_{\max }$ is the maximum specific growth rate of the organism and $K_{\mathrm{s}}$, the saturation constant, is a measure of the organism's affinity for the limiting substrate; $K_{\mathrm{s}}$ is the substrate concentration at which the organism grows at half $\mu_{\max }$. Eqn (1) is of the same form as the MichaelisMenten equation used to describe enzyme kinetics. Thus, growth in a chemostat is limited by the rate of transport of a particular substrate into the organism and this transfer usually follows unidirectional Michaelis-Menten kinetics (Van Uden, 1969). The development of chemostats (Novick \& Szilard, 1950a) made it possible to study the growth of micro-organisms under steady-state conditions and to study the effects of individual environmental parameters on their physiology (James, 1961). However, although environmental parameters can be maintained constant in a chemostat, if cultivation is prolonged, the 
microbial population adapts to its environment by mutation and natural selection. Any mutation conferring a selective advantage to a member of the population, causes it to accumulate in the chemostat vessel and eventually replace all or most of the parental strain (Moser, 1958; Powell, 1958). Thus, microbial populations in a chemostat are not static, but are constantly evolving.

In chemostat cultures, the competitive advantage of one strain relative to another can be quantified by calculating the selection coefficient $(s)$ :

$$
s=\frac{\ln \left[\frac{p_{(t)}}{q_{(t)}}\right]-\ln \left[\frac{p_{(0)}}{q_{(0)}}\right]}{t}
$$

where $p_{(t)}$ is the concentration of one strain at time $t, q_{(t)}$ is the concentration of the second strain at time $t$, and $p_{(0)}$ and $q_{(0)}$ are the initial concentrations of each strain (Dykhuizen \& Hartl, 1981). If the selection coefficient for one strain is positive, it has a selective advantage over the other strain; a negative selection coefficient indicates a selective disadvantage.

Selective advantages for mutants appearing in chemostat cultures have been divided into two main categories: mutants which have a higher maximum specific growth rate $\left(\mu_{\max }\right)$ than the parental strain and mutants which have a lower saturation constant $\left(K_{\mathrm{s}}\right)$ for the limiting nutrient than the parental strain (Moser, 1958; Dykhuizen \& Hartl, 1983). $\mu_{\max }$ mutants are usually selected at high dilution rates and $K_{\mathrm{s}}$ mutants are usually selected at low dilution rates. Using chemostat cultures grown at low dilution rates, Novick \& Szilard (1950b) isolated a mutant of Escherichia coli with improved uptake of tryptophan, and Adams et al. (1985) isolated mutants of Saccharomyces cerevisiae with improved glucose uptake. However, apart from a mutant of $F$. graminearum with a possible improvement in magnesium uptake (Wiebe et al., 1992), $K_{\mathrm{s}}$ mutants of filamentous fungi have not been isolated from chemostat cultures. Similary, using chemostat cultures at high dilution rates, Helling et al. (1987) isolated E. coli mutants with higher $\mu_{\max }$ values than the parental strain: Penicillium cbrysogenum, Aspergillus nidulans and $F$. graminearum mutants with higher $\mu_{\max }$ values than their parental strains have been isolated by Righelato (1976), Carter \& Bull (1969) and Wiebe et al. (1992), respectively.

In contrast to advantageous mutations, neutral mutations (i.e. those which confer neither a selective advantage nor disadvantage to the mutant relative to the parental strain) accumulate very slowly in the population (maximally at the forward mutation rate) and never attain high concentrations (Novick \& Szilard, 1950b; Moser, 1958) unless they are linked to an advantageous mutation. Novick \& Szilard (1950b) observed the accumulation of neutral mutations (resistance to T5 bacteriophage) in chemostat populations of $E$. coli. These workers observed that periodic decreases in the T5-resistant mutant population occurred if the culture was maintained in the chemostat long enough. These decreases occurred when a new T5-sensitive strain with a selective advantage re- placed the T5-sensitive parental strain and the T5-resistant mutant. This phenomenon is now referred to as periodic selection (Novick \& Szilard, 1950b; Dykhuizen \& Hartl, 1983 ) and has been observed subsequently in populations of S. cerevisiae (Paquin \& Adams, 1983a) and F. graminearum (Wiebe et al., 1993). It is possible to study periodic selection in F. graminearum because it produces macroconidia (Wiebe \& Trinci, 1991) which, although multinucleate, are homokaryotic (Miller, 1946). Since macroconidia are formed from uninucleate phialides, the nuclei in macroconidia harvested from a culture provide a sample of the nuclei present in the mycelial biomass. Thus, periodic selection can be followed in F. graminearum by monitoring neutral mutations occurring in macroconidia.

In this paper we describe periodic selection in the Quorn ${ }^{\circledR}$ myco-protein fungus, $F$. graminearum A3/5 grown in a glucose-limited chemostat culture at a dilution rate of $0.05 \mathrm{~h}^{-1}$ (F. graminearum has a $D_{\text {crit }}$ of about $0 \cdot 23 \mathrm{~h}^{-1}$ in this medium) and the isolation of mutants with improved glucose uptake ( $K_{\mathrm{s}}$ mutants).

\section{METHODS}

Organism and medium. Fusarium graminearum Schwabe strain A3/5 was obtained from Mr T. W. Naylor, Marlow Foods, Billingham, UK. Stock cultures were maintained as macroconidia at $-70{ }^{\circ} \mathrm{C}$ in $20 \%(\mathrm{v} / \mathrm{v})$ glycerol. Inocula were prepared as described by Wiebe $e$ al. (1991).

The defined medium of Vogel (1956) was used with glucose as the carbon source instead of sucrose. For submerged cultures, $1.65 \mathrm{~g}\left(\mathrm{NH}_{4}\right)_{2} \mathrm{SO}_{4} \mathrm{l}^{-1}$ was substituted for $2 \mathrm{~g} \mathrm{NH}_{4} \mathrm{NO}_{3} \mathrm{l}^{-1}$ as the nitrogen source. Vogel's mineral salts solution was prepared at $50 \times$ final concentration, sterilized by membrane $(0.2 \mu \mathrm{m}$ diam. pore size) filtration and added to the sterile glucose solution. Glucose solutions [final concentration of $3 \mathrm{~g}$ glucose $(1 \mathrm{me}-$ dium $)^{-1}$ ] for chemostat cultures were prepared in 101 volumes and autoclaved for $60 \mathrm{~min}$ at $121^{\circ} \mathrm{C}$. No caramelization and no significant loss of glucose were observed. For plate cultures, modified Vogel's medium, containing $10 \mathrm{~g}$ glucose $\mathrm{l}^{-1}$ and $2 \mathrm{~g}$ $\mathrm{NH}_{4} \mathrm{NO}_{3} \mathrm{I}^{-1}$ was solidified with agar (Davis Gelatine; $15 \mathrm{~g} \mathrm{l}^{-1}$, final concentration). For media to detect resistant mutants, cycloheximide $(250 \mu \mathrm{M})$ or potassium chlorate $(300 \mathrm{mM})$ was added to modified Vogel's medium. All cultures were incubated at $25^{\circ} \mathrm{C}$.

Chemostat cultures. Cultures were grown in a Braun Biostat $\mathrm{M}$ (2 l) fermenter (B. Braun Medical Ltd.) as described by Wiebe \& Trinci (1991). Biomass retention in the fermenter vessel was monitored by taking dry weight measurements of culture samples both from inside the fermenter vessel and from the overflow. No retention of biomass in the vessel was observed.

Monitoring of cycloheximide-resistant mutants and highly branched (colonial) mutants. Samples (about $10 \mathrm{ml}$ ) were removed daily from the fermenter vessel and cycloheximide resistance was monitored in the macroconidial population. Macroconidia were separated from the mycelial biomass by filtering the culture suspension through two layers of sterile lens tissue (Whatman No. 105) and viable counts were made on modified Vogel's medium solidified with agar (10 replicate plates per sample), as described by Wiebe et al. (1991). Approximately $3 \times 10^{4}$ macroconidia per plate were also in- 


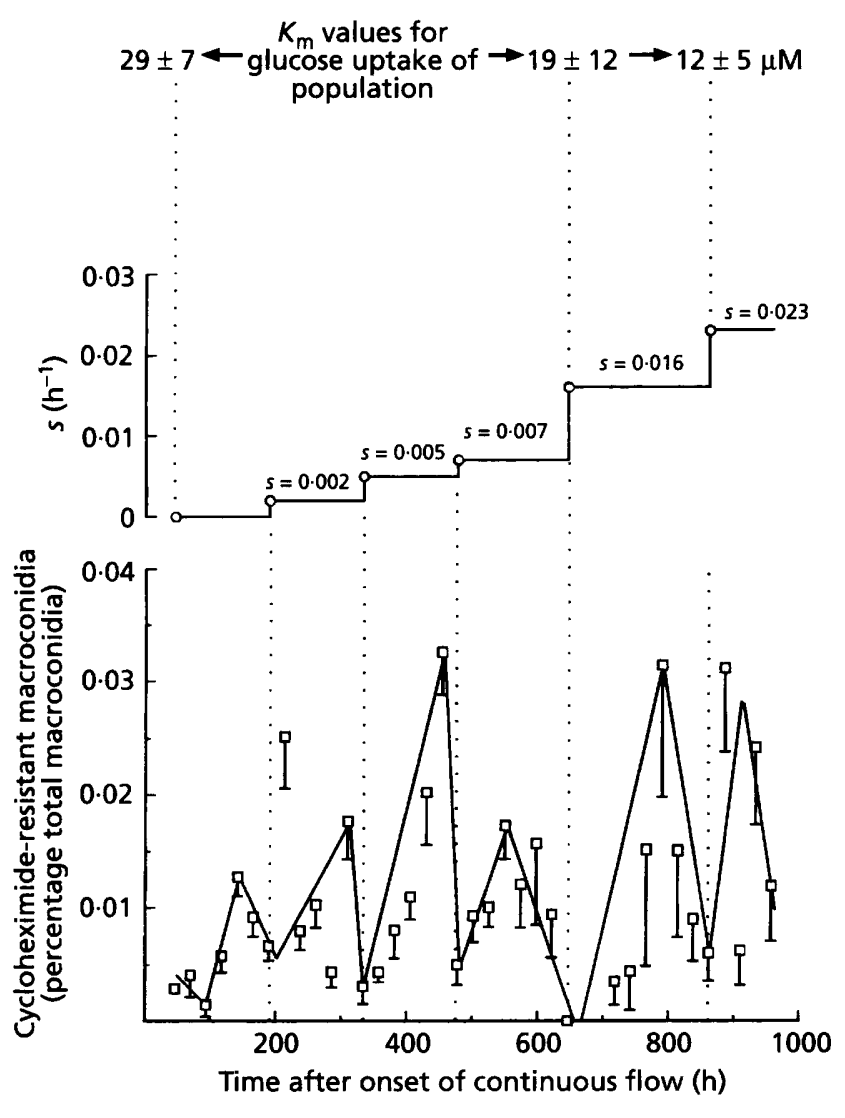

Fig. 1. Fluctuations in the proportion of cycloheximide-resistant macroconidia in a population of $F$. graminearum grown in a glucose-limited chemostat culture $\left(25^{\circ} \mathrm{C}, \mathrm{pH} 5.8 \pm 0.1\right)$ at a dilution rate of $0.05 \mathrm{~h}^{-1}$. The lines indicate increases and decreases in the cycloheximide-resistant population, following the principle that three or more consecutively increasing or decreasing data points indicate the trends in the population. The upper part of the figure gives selection coefficients (s), relative to strain $A 3 / 5$ (chlorate-resistant), of populations derived from samples taken at 190, 334, 478, 646 and $862 \mathrm{~h}$ after the onset of continuous medium flow in a chemostat. The error bars give the SEM.

oculated onto modified Vogel's medium containing $250 \mu \mathrm{M}$ cycloheximide (10 replicate plates per sample). The plates were incubated for 3 (for viable counts) or $6 \mathrm{~d}$ (to detect cycloheximide resistance).

If present, highly branched (so-called colonial) mutants could have been identified from the appearance of their colonies (Wiebe et al., 1991) on Vogel's $\mathrm{NH}_{4} \mathrm{NO}_{3}$ agar-solidified medium

Isolation and 'marking' of mutants. Stock plates were inoculated with $0.1 \mathrm{ml}$ of culture samples (mycelia plus macroconidia) at 190,344,478, 646 and $862 \mathrm{~h}$ after the onset of continuous flow (Fig. 1); plates were incubated at $25{ }^{\circ} \mathrm{C}$ for at least $8 \mathrm{~d}$ before storage at $4{ }^{\circ} \mathrm{C}$. Macroconidia harvested from these stock plates and chlorate-resistant A3/5 macroconidia were subsequently used to inoculate chemostat cultures to measure selection coefficients between the evolved chemostat population and A3/5 (Figs 1 and 2). Although macroconidia harvested from the stock plates gave rise to a mixed population, a substantial proportion would consist of the advantageous mutant which displaced the previous strain. Mutant A28-S was isolated from macroconidia harvested from a stock plate inoculated with a sample taken from the chemostat at $862 \mathrm{~h}$ (Fig. 1). Chlorate-resistant mutants of strains A3/5 and A28-S were selected by inoculating agar-solidified modified Vogel's medium containing $300 \mathrm{mM}$ potassium chlorate with about $5 \times 10^{4}$ macroconidia and chlorate-resistant colonies were isolated after 4-6 d incubation. Each isolate was subcultured onto Vogel's medium containing $\mathrm{NaNO}_{3}$ as the sole nitrogen source, as well as onto Vogel's medium containing $\mathrm{NH}_{4} \mathrm{NO}_{3}$. Only nitrate-non-utilizing strains were retained for competition experiments. Chlorate-resistant mutants of $F$. graminearum A3/5 are very stable, with a reversion frequency of less than 1 in $10^{7}$ macroconidia (G. D. Robson, unpublished results).

Measurement of selection coefficients. Selection coefficients (eqn 2) were determined using chemostat cultures inoculated with a mixture of macroconidia harvested from a chlorateresistant (nitrate-non-utilizing) strain and a chlorate-sensitive (nitrate-utilizing) strain (Fig. 2). Chlorate resistance confers neither a selective advantage nor a disadvantage to the labelled strain when ammonium is used as the nitrogen source in the medium. Competitions between A3/5 and A28-S at a dilution rate of $0.05 \mathrm{~h}^{-1}$ were repeated using chlorate-resistant mutants of each strain to demonstrate the selective neutrality of chlorate resistance in the culture conditions used. Competitions between $\mathrm{A} 3 / 5$ and $\mathrm{A} 28 \mathrm{~S}$ at dilution rates above $0.05 \mathrm{~h}^{-1}$ were made using chlorate-sensitive A3/5 and chlorate-resistant A28-S. Competitions between $\mathrm{A} 3 / 5$ and the evolved chemostat populations were made using chlorate-resistant A3/5 and chloratesensitive isolates. Counts of viable colony forming units (derived from either mycelial fragments or macroconidia) were made on modified Vogel's medium with $\mathrm{NH}_{4} \mathrm{NO}_{3}$ as the nitrogen source, with $\mathrm{NaNO}_{3}$ as the nitrogen source, and with $\mathrm{NH}_{4} \mathrm{NO}_{3}$ plus $300 \mathrm{mM}$ potassium chlorate (10 replicate plates per sample for each medium). The plates were incubated for $3 \mathrm{~d}$ and the proportion of chlorate-resistant (nitrate-non-utilizing) colonies in the total population was determined. The proportion of nitrate-utilizing colonies in the total population was determined independently.

Glucose transport. $K_{m}$ values for glucose uptake were measured (in duplicate) as described by Wiebe et al. (1992). Uptake was followed for $150 \mathrm{~s}$ after the addition of $10-50 \mu \mathrm{M}$ (final concentration) glucose $\left[0.5 \mu \mathrm{Ci}(18.5 \mathrm{kBq})\left[{ }^{14} \mathrm{C}\right]\right.$ glucose $\mu \mathrm{M}^{-1}$, specific activity $290 \mathrm{mCi}(10.7 \mathrm{MBq}) \mathrm{mmol}^{-1}$, Amersham]. Uptake of glucose (at an initial concentration of $200 \mu \mathrm{M}$ ) was also measured in the presence of $2 \mathrm{mM}$ mannose. $K_{\mathrm{m}}$ values for glucose uptake were calculated using the Multifit program of Walmsley \& Lowe (1985).

Measurements of mycelial morphology. Hyphal length (the longest hypha in a mycelial fragment) and hyphal growth unit length (a measure of hyphal branching; Trinci, 1974) were measured using a MeasureMouse graphics system (Analytical Measuring Systems) and an Amstrad PC1512 connected to a Nikon microscope (Wiebe \& Trinci, 1991). As the majority of mycelia were unbranched or very sparsely branched, hyphal growth unit length measurements (made on mycelia with six or more branches) were not necessarily representative of the total population.

\section{RESULTS}

\section{Periodic selection}

A chemostat culture of $F$. graminearum A3/5 was grown for $957 \mathrm{~h}$ or 69 generations at a dilution rate of $0.05 \mathrm{~h}^{-1}$ (doubling time of $13.9 \mathrm{~h}$ ). Six oscillations in the con- 


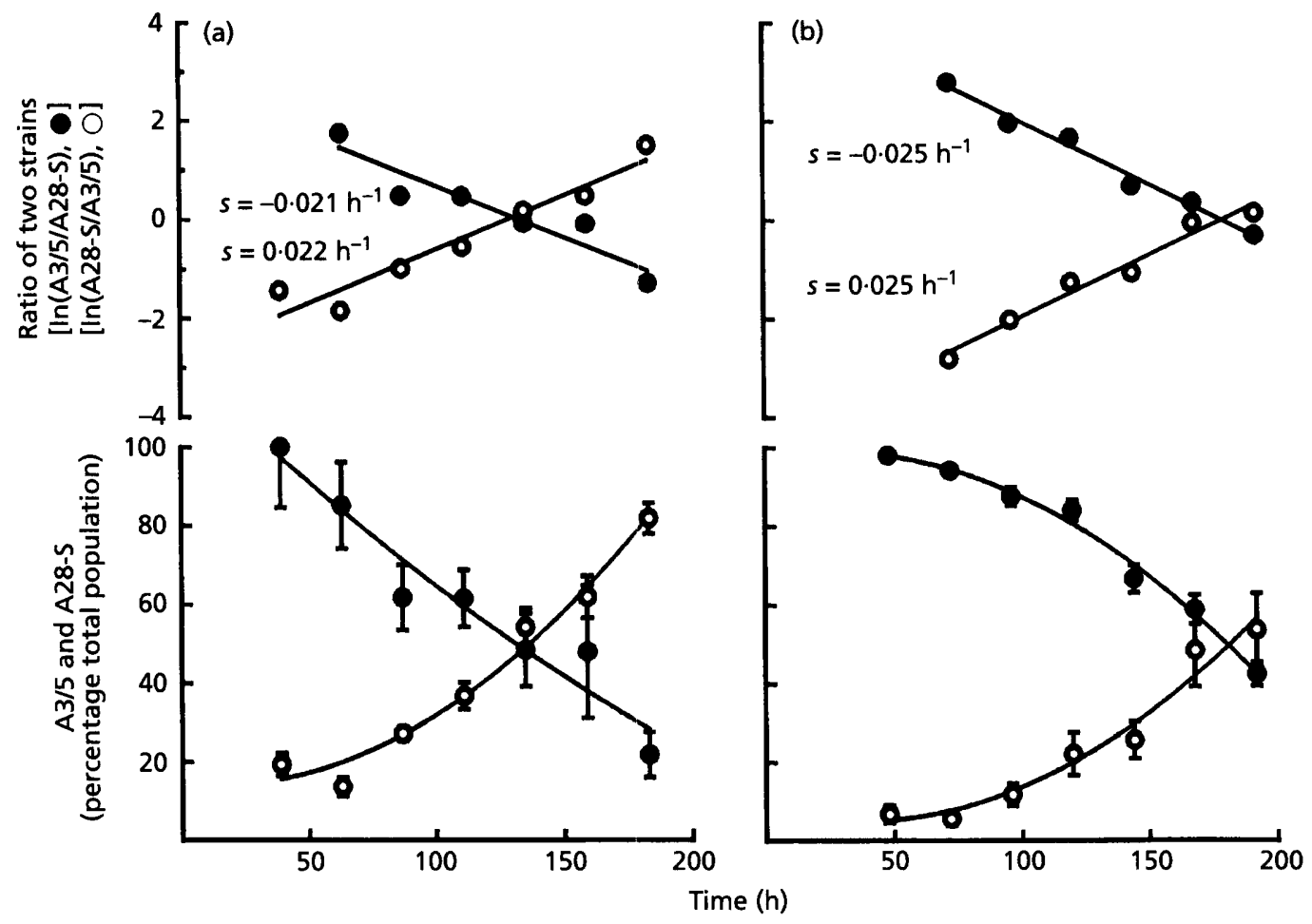

Fig. 2. Competitions between $F$. graminearum strains $A 3 / 5(O)$ and $A 28-S(O)$ grown in glucose-limited chemostat culture at a dilution rate of $0.05 \mathrm{~h}^{-1}$. (a) Chlorate-resistant A3/5. (b) Chlorate-resistant A28-S. The proportion of chlorateresistant propagules and of nitrate-utilizing propagules in the total population were determined separately for each sample.

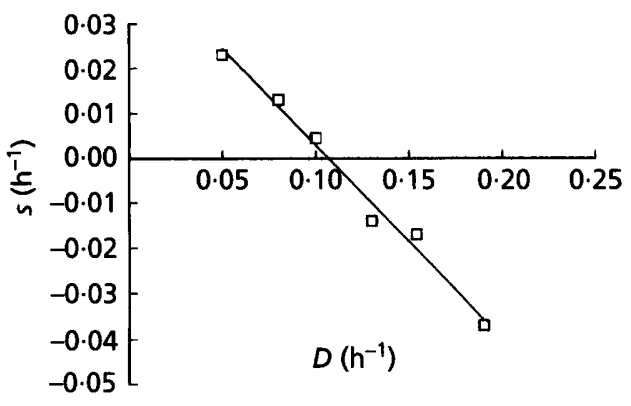

Fig. 3. Selection coefficients (s) for F. graminearum strain A28-S relative to strain $A 3 / 5$ in glucose-limited chemostat culture at dilution rates $(D)$ between $0.05 \mathrm{~h}^{-1}$ and $0.19 \mathrm{~h}^{-1}$.

centration of cycloheximide-resistant macroconidia in the chemostat were observed, with an average $( \pm \mathrm{SE})$ interval of $148 \pm 22 \mathrm{~h}$ (about 11 generations) between peaks (Fig. 1). The mutation rate from cycloheximide sensitivity to cycloheximide resistance was estimated (from the slopes of the six increases in cycloheximide resistance observed during the fermentation) to be $1 \cdot 8( \pm 0 \cdot 2) \times 10^{-6} \mathrm{~h}^{-1}$, or $2.5 \times 10^{-5}$ generation $^{-1}$.

Each decrease observed in the concentration of cycloheximide-resistant macroconidia suggested that a pre- viously successful strain was replaced by a new, cycloheximide-sensitive strain which was more competitive than the previous strain. The new strain comprised an increasing proportion of the population until it also was replaced by an even more competitive strain (Fig. 1). The competitive advantage of the strain present at the start (Fig. 1) of each increase in the cycloheximide-resistant population of macroconidia was determined relative to A3/5 by growing them in mixed culture in a glucoselimited chemostat at $0.05 \mathrm{~h}^{-1}$ (see Fig. 2 for A28-S competition). The selective advantage of the first mutant population was too small $\left(s=0.002 \mathrm{~h}^{-1}\right)$ to be measured accurately, but a progressive increase in selection coefficient was observed for each of the subsequent mutant populations with the most evolved population having a selection coefficient of $0.023 \mathrm{~h}^{-1}$ (Fig. 1).

\section{Characterization of strain A28-S as a $\boldsymbol{K}_{\mathrm{s}}$ mutant}

A strain (designated A28-S) isolated $862 \mathrm{~h}$ after the onset of continuous flow had a selection coefficient of $0.023 \mathrm{~h}^{-1}$ when grown in a glucose-limited chemostat at a dilution rate of $0.05 \mathrm{~h}^{-1}$ (Figs 1 and 2). However, A28-S progressively lost its selective advantage over $\mathrm{A} 3 / 5$ when the dilution rate was increased from 0.05 to $0 \cdot 11 \mathrm{~h}^{-1}$, and at dilution rates above $0.11 \mathrm{~h}^{-1}$ it was at a selective disadvantage relative to $\mathrm{A} 3 / 5$ (Fig. 3 ). When grown at a dilution rate of $0.05 \mathrm{~h}^{-1}$ in mannose-, fructose- or xylose- 
Table 1. Selection coefficients of $F$. graminearum strain A28-S, relative to strain $A 3 / 5$, in chemostat cultures at a dilution rate of $0.05 \mathrm{~h}^{-1}\left(25^{\circ} \mathrm{C}, \mathrm{pH} \mathrm{5.8 \pm 0.1)}\right.$ which were limited for various carbon sources

\begin{tabular}{|lc|}
\hline $\begin{array}{l}\text { Limiting } \\
\text { carbon } \\
\text { source }\end{array}$ & $\begin{array}{c}\text { Selection } \\
\text { coefficient } \\
\left(\boldsymbol{s}, \mathbf{h}^{-\mathbf{1}}\right)\end{array}$ \\
\hline Xylose & 0.025 \\
Glucose & 0.023 \\
Fructose & 0.022 \\
Mannose & 0.020 \\
Ribose & 0.013 \\
Maltose & 0.011 \\
\hline
\end{tabular}

limited chemostat cultures, A28-S had a selective advantage over $A 3 / 5$ (Table 1 ). However, compared with glucose-limited cultures, the selective advantage of A28-S relative to $\mathrm{A} 3 / 5$ was considerably reduced in maltose- and ribose-limited chemostat cultures grown at $0.05 \mathrm{~h}^{-1}$ (Table 1).

AC28-\#26 (the strain isolated from the penultimate adaptive peak) and A28-S had $K_{\mathrm{m}}$ values for glucose uptake of $19 \pm 4$ and $12 \pm 5 \mu \mathrm{M}$, respectively, compared with $29 \pm 7 \mu \bar{M}$ for the $\bar{K}_{\mathrm{m}}$ for glucose uptake by A3/5 (Wiebe et al., 1992). Glucose uptake by $F$. graminearum A3/5 was reduced to $63 \%$ of the control when $2 \mathrm{mM}$ mannose was included in the reaction mixture. Addition of $2 \mathrm{mM}$ unlabelled glucose to the reaction mixture resulted in an $85 \%$ reduction in glucose uptake.

\section{Sporulation and morphology}

F. graminearum A3/5 in a glucose-limited culture grown at a dilution rate of $0.05 \mathrm{~h}^{-1}$ initially produced about $1 \times 10^{6}$ macroconidia $\mathrm{ml}^{-1}$ but this decreased with time until at $800 \mathrm{~h}$ after the onset of continuous flow the culture contained only about $1 \times 10^{4}$ macroconidia $\mathrm{ml}^{-1}$ (Fig. 4). When $\mathrm{A} 3 / 5$ was grown in glucose-limited chemostat culture at a dilution rate of $0.06 \mathrm{~h}^{-1}$ the population had a mean hyphal length (the longest hypha in a fragment) of $502 \pm 9 \mu \mathrm{m}$ (mean $\pm \mathrm{SE})$ and a hyphal growth unit length of $94 \pm 7 \mu \mathrm{m}$ (Wiebe \& Trinci, 1991). However, at the end of the present fermentation, the population had a mean hyphal length of $1104 \pm 69 \mu \mathrm{m}$ (100 replicates) and a mean hyphal growth unit of $238 \pm 15 \mu \mathrm{m}$ ( 25 replicates). Thus, mycelial morphology changed during the experimental period. No highly branched (colonial) mutants (Trinci, 1992) were observed in the population at any time during the $957 \mathrm{~h}$ fermentation period.

\section{Further evolution of $\boldsymbol{F}$. graminearum in a second chemostat}

A second chemostat was inoculated with macroconidia harvested from stock plates inoculated with a culture

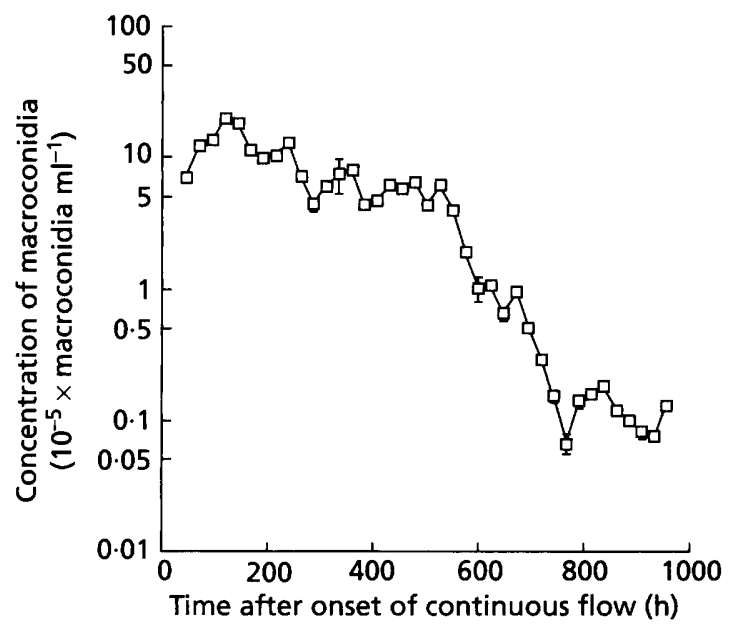

Fig. 4. Concentration of macroconidia in a population of $F$. graminearum grown in a glucose-limited chemostat culture at a dilution rate of $0.05 \mathrm{~h}^{-1}\left(25^{\circ} \mathrm{C}, \mathrm{pH} 5.8 \pm 0.1\right)$.

sample from the first chemostat. No colonial mutants were present after a further $1345 \mathrm{~h}$ of glucose-limited growth at a dilution rate of $0 \cdot 05 \mathrm{~h}^{-1}$. The total evolutionary time of the combined chemostat cultures was $2207 \mathrm{~h}$ or about 159 generations.

\section{DISCUSSION}

The changes observed in the concentration of cycloheximide-resistant macroconidia in the chemostat population suggested that four advantageous mutants had accumulated and been eliminated from the population before the A28-S mutant appeared (Fig. 1). Measurement of selection coefficients of these mutants, relative to A3/5 demonstrated that each mutant population which arose had a higher selective advantage than all the previous mutant populations (Fig. 1). This contrasts with the results of Paquin \& Adams (1983b) who found that evolving yeast populations did not show additive improvements, although each successive strain had a selective advantage relative to its immediate precursor. However, since the five mutants detected (Fig. 1) may have arisen separately during batch culture (Wiebe et al., 1993; Dykhuizen \& Hartl, 1983), A28-S may not carry five mutations. From the time of the last cycloheximide peak and from the selection coefficient of A28-S observed, it was estimated that the presence of about 8-80 mutant propagules in the population at the end of batch growth $\left(8 \times 10^{9}\right.$ propagules) would account for the appearance of A28-S at $886 \mathrm{~h}$ after the onset of continuous flow (Wiebe et al., 1993).

A28-S displaced $A 3 / 5$ at slow dilution rates $(0.05$ to $\left.0 \cdot 10 \mathrm{~h}^{-1}\right)$ in glucose-limited cultures but, as predicted for a $K_{\mathrm{s}}$ mutation, the selection coefficient decreased with increase in dilution rate (Fig. 3). However, as the selection coefficient of A28-S relative to A3/5 actually became 
negative at dilution rates above $0 \cdot 11 \mathrm{~h}^{-1}$, A28-S may have a reduced $\mu_{\max }$ compared to A3/5. A28-S retained its selective advantage over $\mathrm{A} 3 / 5$ when grown in a mannoselimited chemostat-as predicted for a mutant with improved glucose uptake (both sugars are taken up by the same transport system). However, the selective advantage of A28-S over A3/ 5 was not totally eliminated in maltoseor ribose-limited chemostat cultures and was not reduced in fructose- or xylose-limited chemostat cultures (Table 1). Unlike mannose, the latter sugars are not taken up by the glucose transport system (Wiebe et al., 1992). Therefore, A28-S may have an altered carbon metabolism as well as a reduced $K_{\mathrm{m}}$ for glucose. The former type of mutant of $F$. graminearum has been isolated previously (Wiebe et al., 1992).

The mutation rate for cycloheximide resistance was estimated to be $2.5 \times 10^{-5}$ generation $^{-1}$ or $1 \cdot 8( \pm 0 \cdot 2) \times$ $10^{-6} \mathrm{~h}^{-1}$. At least four loci for cycloheximide resistance have been identified in Neurospora crassa (Vomvoyanni, 1974), so it is likely that, if a similar number of loci are involved in cycloheximide resistance in F. graminearum, the mutation rate for individual genes may be less than $6.3 \times 10^{-6}$ generation $^{-1}$. Further, modifier genes, which control the level of cycloheximide resistance, also occur in Neurospora crassa (Vomvoyanni, 1974; Rothschild et al., 1975). Mutation rates for filamentous organisms are difficult to determine using batch cultures, and therefore measurements of mutations in filamentous fungi have been limited to estimating mutation frequency. Spontaneous mutation frequencies of between $1 \times 10^{-4}$ and $1 \times 10^{-8}$ per conidium have been reported for Neurospora crassa (Fincham et al., 1979). These frequencies do not include a time element, and therefore are not strictly comparable to mutation rates estimated here. Paquin \& Adams (1983a) observed a mutation rate of about $1 \times 10^{-7}$ generation $^{-1}$ in a diploid yeast population grown in a glucose-limited chemostat at a dilution rate of about $0 \cdot 20 \mathrm{~h}^{-1}$, a rate lower than that observed for $F$. graminearum. However, as the yeast was diploid, mutation of the recessive modifier genes (Rothschild et al., 1975) would not have been detected unless rendered homozygous by a subsequent gene conversion event, whereas both dominant and recessive mutations would be detected in homokaryotic macroconidia of $F$. graminearum.

When grown in a glucose-limited chemostat at a dilution rate of $0.05 \mathrm{~h}^{-1}$, A28-S produced fewer macroconidia and much more sparsely branched fragments than $A 3 / 5$. In four unperturbed glucose-limited fermentations of $F$. graminearum A3/5 grown at a dilution rate of $0 \cdot 18$ $0.19 \mathrm{~h}^{-1}$, highly branched (colonial) mutants were observed in the population 360-447 h (99-115 generations) after the onset of continuous flow. However, as no highly branched (colonial) mutant was observed in glucoselimited chemostat cultures grown at a dilution rate of $0.05 \mathrm{~h}^{-1}$ after 159 generations, we conclude that there is a lower probability of such mutants appearing in glucoselimited populations of $F$. graminearum $A 3 / 5$ at low $\left(0.05 \mathrm{~h}^{-1}\right)$ than at high $\left(0.19 \mathrm{~h}^{-1}\right)$ dilution rates. Thus, increased hyphal branching does not appear to be associated with selective advantages of mutants selected at low dilution rates in glucose-limited chemostat cultures of F. graminearum.

Wiebe et al. (1992) showed that a mutation in an enzyme of the pentose phosphate pathway in F. graminearum A3/5 resulted in a colonial mutant with a selective advantage in glucose-limited cultures at high dilution rates. The present results suggest that strain A28-S had an altered carbon metabolism as well as an improved glucose transport system. Fungal evolution has so far been studied at high (Trinci, 1992; Withers et al., 1994) and low dilution rates. By using chemostats at intermediate dilution rates it should be possible to isolate a range of strains with both biochemical and morphological mutations.

\section{ACKNOWLEDGEMENTS}

We thank Mr G. Grange and Mr N. Poole for technical assistance and Marlow Foods for financial support.

\section{REFERENCES}

Adams, J., Paquin, C., Oeller, P. W. \& Lee, L. W. (1985). Physiological characterization of adaptive clones in evolving populations of the yeast, Saccharomyces cerevisiae. Genetics 110, 173-185.

Carter, B. L. A. \& Bull, A. T. (1969). Studies of fungal growth and intermediary carbon metabolism under steady and non-steady state conditions. Biotechnol Bioeng 11, 785-804.

Dykhuizen, D. E. \& Hartl, D. (1981). Evolution of competitive ability in Escherichia coli. Evolution 35, 581-594.

Dykhuizen, D. E. \& Hartl, D. L. (1983). Selection in chemostats. Microbiol Rev 47, 150-168.

Fincham, J. R., Day, P. R. \& Radford, A. (1979). Fungal Genetics. London: Blackwell Scientific Publications.

Helling, R. B., Vargas, C. N. \& Adams, J. (1987). Evolution of Escherichia coli during growth in a constant environment. Genetics 116, 349-358.

James, T. W. (1961). Continuous culture of micro-organisms. Ann Rev Microbiol 15, 27-46.

Miller, J. J. (1946). Cultural and taxonomic studies on certain Fusaria. I. Mutations in culture. Can J Res 24 (C), 188-212.

Monod, J. (1942). Recherches sur la Croissance des Cultures Bacteriènnes. Paris: Hermann et Cie.

Moser, H. (1958). The dynamics of bacterial populations maintained in the chemostat. Washington, DC: Carnegie Institute of Washington, Publication 61.

Novick, A. \& Szilard, L. (1950a). Description of the chemostat. Science 112, 715-716.

Novick, A. \& Szilard, L. (1950b). Experiments with the chemostat on spontaneous mutation of bacteria. Proc Natl Acad Sci USA 36, 708-719.

Paquin, C. \& Adams, J. (1983a). Frequency of fixation of adaptive mutations is higher in evolving diploid than haploid yeast populations. Nature 302, 495-500.

Paquin, C. \& Adams, J. (1983b). Relative fitness can decrease in evolving asexual populations of Saccharomyces cerevisiae. Nature 306, 368-371.

Powell, E. O. (1958). Criteria for the growth of contaminants and mutants in continuous culture. J Gen Microbiol 18, 259-268. 
Righelato, R. C. (1976). Selection of strains of Penicillium chrysogenum with reduced penicillin yields in continuous cultures. $J$ Appl Chem Biotechnol 26, 153-159.

Rothschild, H., Germershausen, J. \& Suskind, S. R. (1975). Biochemical genetic studies of cycloheximide resistance in Neurospora crassa. Biochem Genet 13, 283-300.

Trinci, A. P. J. (1974). A study of the kinetics of hyphal extension and branch initiation of fungal mycelia. J Gen Microbiol 81, 225-236.

Trinci, A. P. J. (1992). Myco-protein - a twenty-year overnight success story. Mycol Res 96, 1-13.

Van Uden, N. (1969). Kinetics of nutrient-limited growth. Ann Rev Microbiol 23, 473-486.

Vogel, H. J. (1956). A convenient growth medium for Neurospora (Medium N). Microb Genet Bull 13, 42-44.

Vomvoyanni, V. (1974). Multigenic control of ribosomal properties associated with cycloheximide sensitivity in Neurospora crassa. Nature 248, 508-510.

Walmsley, A. R. \& Lowe, A. G. (1985). Multifit: a flexible nonlinear least squares regression program in BAsic. Comp Methods Progr Biomed 21, 113-118.
Wiebe, M. G. \& Trinci, A. P. J. (1991). Dilution rate as a determinant of mycelial morphology in continuous culture. Biotechnol Bioeng 38, 75-81.

Wiebe, M. G., Trinci, A. P. J., Cunliffe, B., Robson, G. D. \& Oliver, S. G. (1991). Appearance of morphological (colonial) mutants in glucose-limited, continuous flow cultures of Fusarium graminearum A3/5. Mycol Res 95, 1284-1288.

Wiebe, M. G. Robson, G. D., Cunliffe, B., Trinci, A. P. J. \& Oliver, S. G. (1992). Nutrient-dependent selection of morphological mutants of Fusarium graminearum A3/5 isolated from long-term continuous flow cultures. Biotechnol Bioeng 40, 1181-1189.

Wiebe, M. G., Robson, G. D., Cunliffe, B., Oliver, S. G. \& Trinci, A. P. J. (1993). Periodic selection in longterm continuous-flow cultures of the filamentous fungus Fusarium graminearum. $J$ Gen Microbiol 139, 2811-2817.

Withers, J. M., Wiebe, M. G., Robson, G. D. \& Trinci, A. P. J. (1994). Development of morphological heterogenicity in glucoselimited chemostat cultures of Aspergillus oryzae. Mycol Res 98, 95-100.

Received 6 May 1994; revised 12 July 1994; accepted 14 July 1994. 